\title{
ПОВЫШЕНИЕ ПРОПУСКНОЙ СПОСОБНОСТИ РАСПРЕДЕЛИТЕЛЬНЫХ ЭЛЕКТРИЧЕСКИХ СЕТЕЙ С ВОЗОБНОВЛЯЕМЫМИ ИСТОЧНИКАМИ ЭНЕРГИИ ПУТЕМ ИХ РЕКОНФИГУРАЦИИ
}

\author{
Черемисин Василий Титович1, \\ cheremisinvt@gmail.com \\ Третьяков Евгений Александрович1, \\ eugentr@mail.ru \\ 1 Омский государственный университет путей сообщения, \\ Россия, 644046, г. Омск, пр. К. Маркса, 35.
}

\begin{abstract}
Актуальность исследования обусловлена необходимостью разработки технических решений по повышению пропускной способности перспективных распределительных электрических сетей железных дорог с возобновляемыми источниками энергии в темпе процессов изменения спроса на электроэнергию.

Цель: повышение пропускной способности перспективных распределительных электрических сетей железных дорог с возобновляемыми источниками энергии путем оптимальной реконфигурации электрических схем.

Объекты: распределительные электрические сети, источники распределенной генерации активной и реактивной мощности, методы оптимизации для решения задач в электроэнергетике, повышение пропускной способности электрической сети. Методы: оптимизационная задача решалась методом ветвей и грании, имитационное моделирование режимов электрических сетей выполнялось в Matlab Simulink.

Результаты. Разработан алгоритм реконфигурации электрической сети для повышения ее пропускной способности на основе решения задач оптимизации нормальных режимов (статическая реконфигурация) и минимизации потребления электроэнергии без расчета установившихся режимов в темпе процессов изменения спроса на электроэнергию (динамическая реконсригурация). Для обеспечения допустимости режимов распределительных электрических сетей предложены подходы к управлению предложением активных (на базе возобновляемых источников) и реактивных мощностей и спросом активных потребителей. Представлены результаты реализации динамической реконфигурации для тестовой электрической схемы без учета управления предложением мощности и спросом активных потребителей, свидетельствующие об обоснованности предлагаемых подходов к повышению пропускной способности электрической сети.
\end{abstract}

\section{Ключевые слова:}

Реконфигурация, распределительные электрические сети, пропускная способность, источники распределенной генерации активной и реактивной мощности, управление спросом.

\section{Введение}

Электросетевой комплекс железных дорог России осуществляет передачу электроэнергии сторонним потребителям в объеме около 40 млрд кВт·ч в год.

Существующие технологии управления режимами в распределительных электрических сетях железных дорог не предполагают возможности изменения их топологии для обеспечения задач по снижению перегрузки и потерь электроэнергии в темпе процессов изменения спроса на электроэнергию в нормальных режимах. Внедрение возобновляемых источников энергии вызывает необходимость разработки специальных технических решений по повышению пропускной способности перспективных распределительных электрических сетей железных дорог.

Задача по оптимизации структуры электрической сети при изменении активных и реактивных мощностей в последние годы широко прорабатывается в зарубежных и отечественных научных кругах.

В целом представленную задачу можно рассматривать как оптимизационную нелинейную смешанного целочисленного программирования [1], которая решается большинством авторов методами, представленными на рис. 1.

Часть исследователей придерживаются эвристических методов решения задачи оптимальной реконфи- гурации электрической сети [2-5], которые позволяют существенно уменьшить вычислительные и временные ресурсы, но получить приближенные решения. Оптимизационная задача комбинаторного типа решается некоторыми авторами с помощью алгоритмов салюта (фейерверка), нейронных сетей, нечетких множеств [6-9] в основном в условиях ограниченной исходной информации.

Эвристические методы решения комбинаторных оптимизационных задач показали свою высокую эффективность в сравнении с классическими методами перебора при значительном количестве аргументов целевой функции, когда определяющим является не точность, а скорость вычислений.

Методы оптимизации, связанные с поиском на графах, также находят все большее применение при решении задач поиска оптимальной топологии электрической сети, в первую очередь из-за своей однозначной разрешимости [10].

Хотя проблема минимального дерева в теории графов хорошо изучена [11], решение представленной задачи не является простой в силу того, что для построения оптимальной топологии необходимо не только минимизировать сопротивления линий распределительной сети, но и потери электроэнергии, определять потоки мощности для всех вариантов 
структуры сети, а также учитывать ограничения параметров режима и эксплуатации, надежность электроснабжения и прочее.

\section{Постановка задачи}

В данном исследовании предлагается решать задачу оптимальной реконфигурации радиальных электрических схем для повышения пропускной способности, ограничения перегрузки и снижения потерь электроэнергии в перспективной системе электроснабжения железных дорог. При этом в качестве критерия оптимизации топологии электрической сети выступает минимальное потребление электроэнергии при эксплуатационных ограничениях по схемам электроснабжения, допустимым параметрам режима, перегрузкам линий и т. д. Применение целевой функции по минимуму потребления электроэнергии вместо традиционного минимума потерь мощности позволяет учесть изменение всех видов потерь электроэнергии в электрических сетях и статические характеристики нагрузок по напряжению. Кроме этого, данная задача при оперативном управлении решается без расчета установившихся режимов.

\section{Теоретическая часть}

Распределительные электрические сети железных дорог 6-10 кВ выполнены разомкнутыми, как прави- ло, по петлевым и комбинированным схемам, поэтому количество вариантов их реконфигурации достаточно ограниченно, в том числе по эксплуатационным режимам.

Задача заключается в том, чтобы определить, какие из выключателей должны быть открыты, чтобы обеспечит наибольшее снижение электропотребления в электрической сети с заданными нагрузками.

Если для всех комбинационных вариантов положений выключателей выполнять полный расчет токораспределения и/или решать оптимизационную нелинейную задачу целочисленного программирования, это окажется неприемлемым для оперативного управления реконфигурацией электрической сети при перегрузке и/или для снижения потерь электроэнергии. Поэтому предлагается задачу реконфигурации разомкнутой распределительной электрической сети разбить на две подзадачи, динамическую и статическую реконфигурацию, и решать их разными методами.

Реконфигурация электрической сети для быстрого снятия перегрузки элементов осуществляется на основе агентного подхода методом ветвей и границ с использованием рекуррентных соотношений для определения потерь электроэнергии для всех участков без полного расчета установившегося режима (в отличие от [12]).

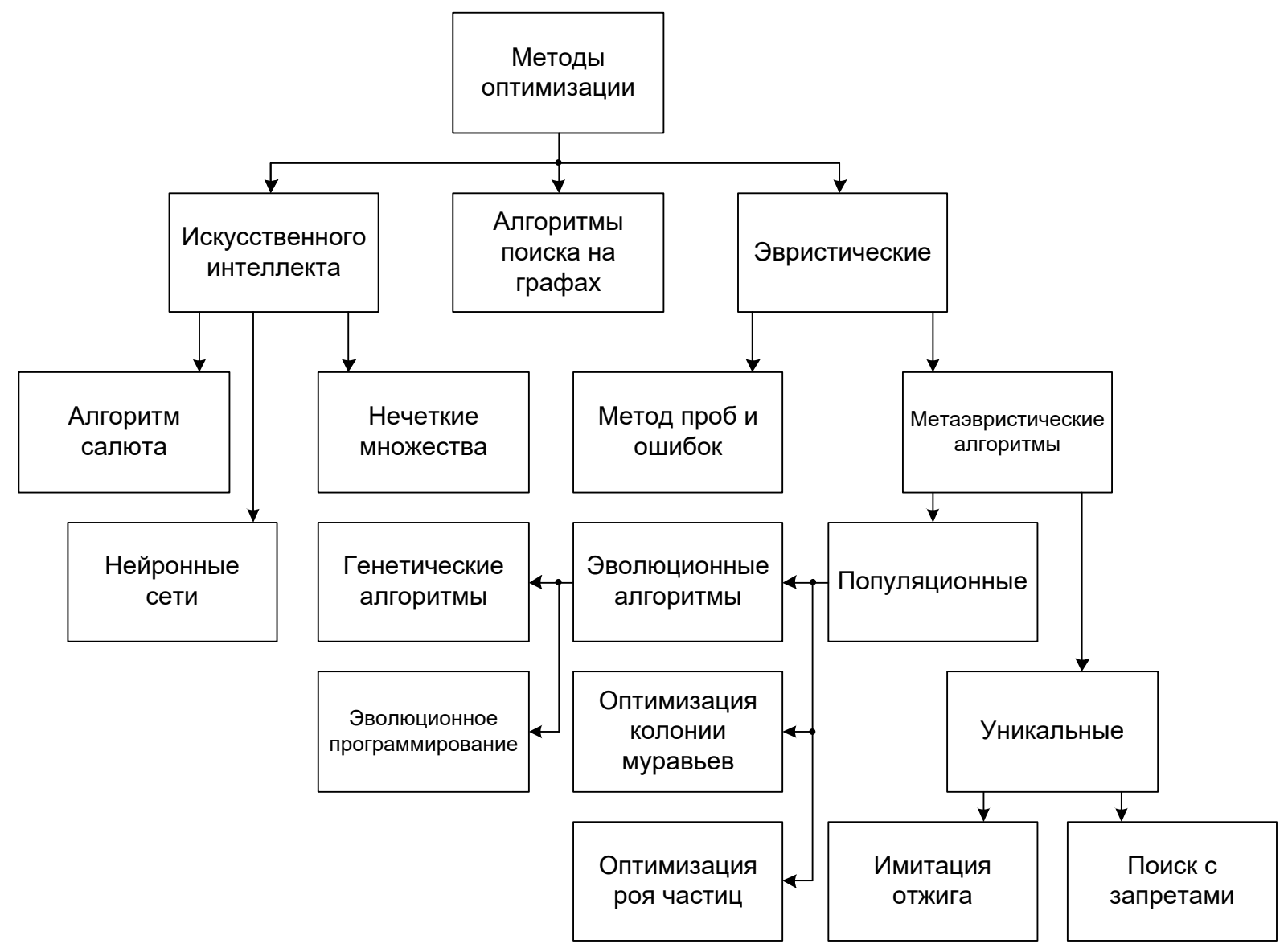

Pис. 1. Методы оптимизации для решения задачи реконфигурации электрической сети

Fig. 1. Optimization methods for solving the problem of electrical network reconfiguration 


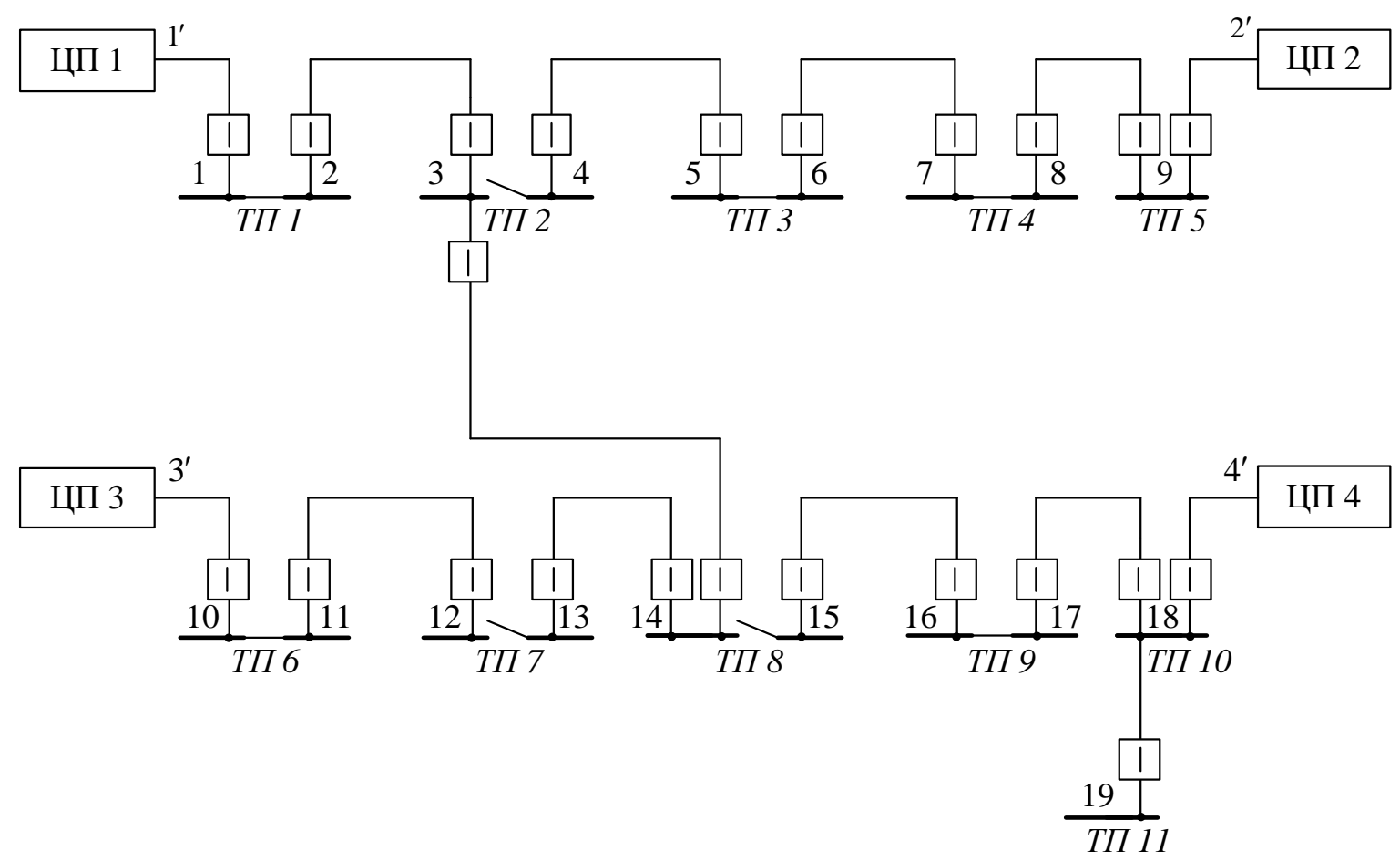

Pис. 2. Схема распределительной электрической сети $10 \kappa B$ железнодорожного узла

Fig. 2. Scheme of $10 \mathrm{kV}$ distribution electric network

В нормальном режиме для выбора топологии электрической сети с минимальным потреблением электроэнергии (с минимальными потерями электроэнергии) решается нелинейная задача оптимизации установившихся режимов, имитирующих реконфигурацию, с учетом прогнозных значений нагрузок.

Представленную на рис. 2 распределительную электрическую сеть с центрами питания (ЦП) (the power center - CР) обозначим в виде графа $G(N ; E)$, где $N$ - шины трансформаторных подстанций (ТП) (the transformer substations - TS): $\mathrm{N}=\{1,2, \ldots, m\}$, a $(i, j),(i, j) \in E$ - линии (дуги, идущие от шины $i$ к шине $j$ ). Данный граф можно разбить на четыре подграфа (по числу центров питания разомкнутой электрической сети): $G_{1}\left(N_{1} ; E_{1}\right), G_{2}\left(N_{2} ; E_{2}\right), G_{3}\left(N_{3} ; E_{3}\right), G_{4}\left(N_{4} ; E_{4}\right)$. При этом $N_{1}(i)=\{j \mid(i, j) \in E\}, N_{2}(i)=\{j \mid(j, i) \in E\}$ и т. д.

Модель потоков мощности для указанной радиальной электрической сети может быть представлена в виде:

$$
\begin{aligned}
& P=\sum_{i \in N} \sum_{j \in N(i)} \delta_{i j}\left(P_{\mathrm{н} j}-P_{\mathrm{rj}}\right)+\sum_{i \in N} \sum_{j \in N(i)} \delta_{i j}\left(\Delta P_{i j}\right) ; \\
& Q=\sum_{i \in N} \sum_{j \in N(i)} \delta_{i j}\left(Q_{\mathrm{rj}}-Q_{\mathrm{rj}}\right)+\sum_{i \in N} \sum_{j \in N(i)} \delta_{i j}\left(\Delta Q_{i j}\right),
\end{aligned}
$$

где $P_{\text {н }}, P_{\text {г }}$ и $Q_{\text {н }}, Q_{\text {г }}$ - активные и реактивные мощности нагрузки и инъекции (от возобновляемых источников энергии) на шине $j ; \Delta P_{i j}=\frac{P_{i j}^{2}+Q_{i j}^{2}}{U_{j}^{2}} r_{i j} ; \Delta Q_{i j}=\frac{P_{i j}^{2}+Q_{i j}^{2}}{U_{j}^{2}} x_{i j}-$ потери активной и реактивной мощности в линиях $i j$; $P_{i j}, Q_{i j}-$ перетоки активной и реактивной мощности в линиях $i j ; r_{i j}, x_{i j}$ - активные и индуктивные сопротивления линий $i j ; \delta_{i j}$ - двоичная переменная $\left(\delta_{i j}=0,1(i, j) \in E\right)$. Основным допущением модели (1) является учет только нагрузочных потерь в линиях.
Для рассматриваемой (рис. 2) разомкнутой электрической сети с четырьмя центрами питания оптимизационная задача динамической реконфигурации на основе (1) может быть записана в виде:

$$
\sum_{\substack{k=1 \\ \delta_{i j}=0,1(i, j) \in E}}^{4} P_{k}\left(\delta_{i j}\right) \rightarrow \min ,
$$

при ограничениях:

$$
\begin{gathered}
\sum_{\substack{j \in N_{1} \\
j \in N_{3}}} P_{i j}-\sum_{\substack{j \in N_{2} \\
j \in N_{4}}} P_{j i}\left\{\begin{array}{l}
\leq P_{i}^{\text {ист }}, i \in N_{1^{\prime}}, N_{2^{\prime}}, N_{3^{\prime}}, N_{4^{\prime}} \\
\leq-\left(P_{\mathrm{rj}}-P_{\text {г }}\right), j \in N_{\mathrm{H}},
\end{array}\right. \\
\sum P_{j}^{\text {ист }}>\sum\left(P_{\text {нј }}-P_{\text {гі }}\right), \Delta P_{i j}=0 ; \\
0 \leq P_{i j}<P_{i j}^{\max } ; 0 \leq Q_{i j}<Q_{i j}^{\max } ; \\
U_{j}^{\min } \leq U_{j}<U_{j}^{\max } ; \\
\sum_{i \neq j} \delta_{i j}=a, a=1, \ldots, n, \delta_{i j}=1 \text { при конкретных } i j .
\end{gathered}
$$

Решение представленной оптимизационной задачи (2) методом ветвей и границ позволит получить значения двоичной переменной, которая описывает состояние ветви графа электрической сети: 0 - линия разомкнута, 1 - линия замкнута.

Определение потоков мощности выполняется без расчета установившегося режима по заданным инъекциям в узлах и топологии электрической сети. Допустимые параметры режима электрической сети по напряжению оцениваются по модулю в конце линии на шине $j$ по данным в начале линии на шине $i$ в виде [13]:

$$
U_{j}=\sqrt{\left(U_{i}-\frac{r_{i j} P_{i}+x_{i j} Q_{i}}{U_{i}}\right)^{2}+\left(\frac{x_{i j} P_{i}-r_{i j} Q_{i}}{U_{i}}\right)^{2}} .
$$




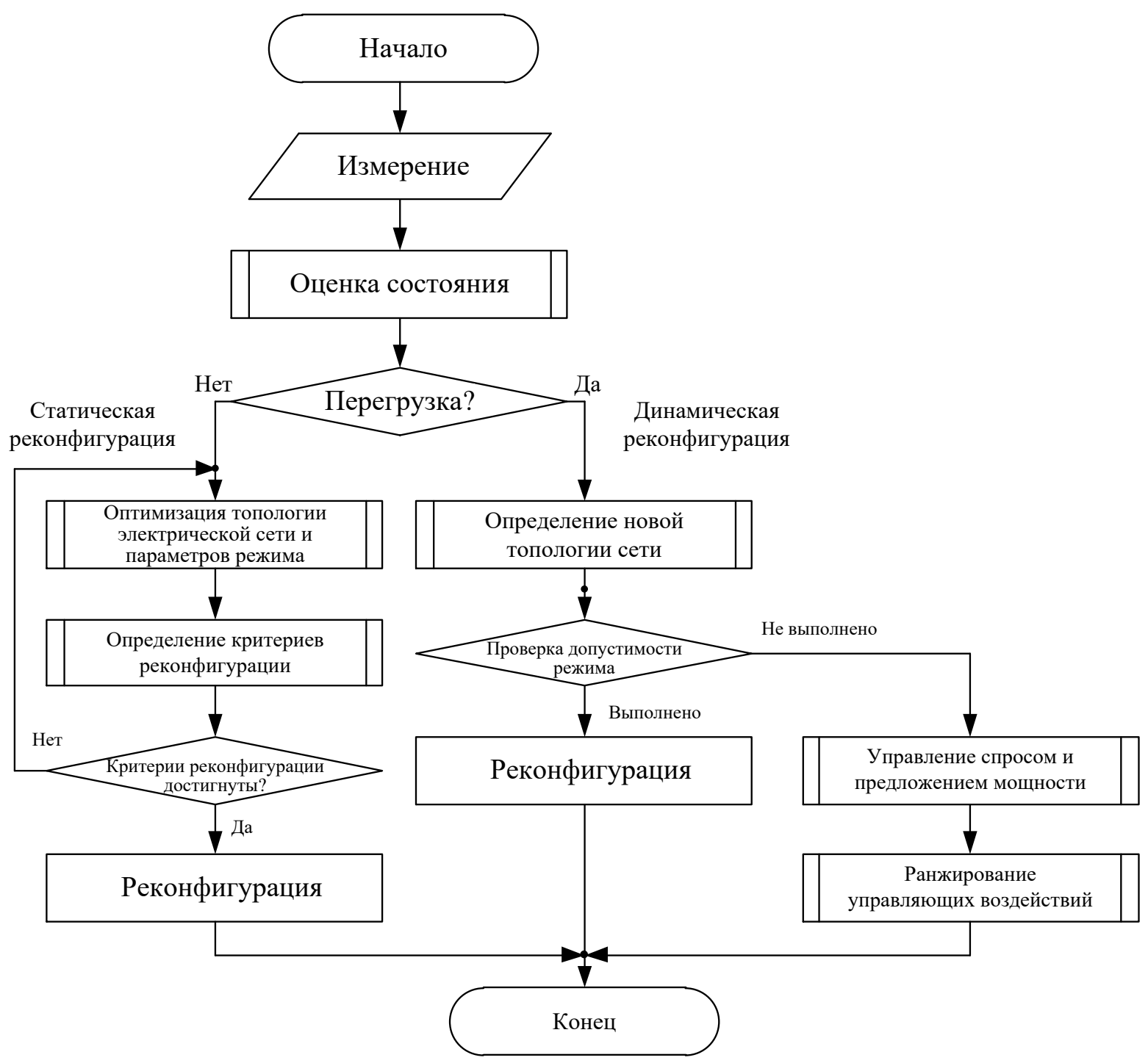

Рис. 3. Алгоритм повышения пропускной способности электрической сети

Fig. 3. Algorithm of increasing the capacity of the electrical network

Реконфигурация электрической сети для исключения перегрузки линий должна обеспечивать допустимый новый режим, если это возможно, иначе - прибегать к управлению предложением и спросом, вплоть до отключения нагрузок.

Критерии реконфигурации:

- обеспечение баланса мощности, включая резерв;

- исключение перегрузки электрооборудования;

- исключение недопустимых уровней напряжения и частоты;

- обеспечение требуемой категории надежности электроснабжения;

- обеспечение требуемой частоты реконфигурации (порог).

Предлагаемый алгоритм рассматриваемой реконфигурации электрической сети представлен на рис. 3.

Система управления реконфигурацией обеспечивает непрерывный контроль и оценку состояния сети, а в случае отсутствия перегрузки элементов электрической сети выполняет оптимизацию новой тополо- гии электрической сети и параметров режима с учетом прогнозных значений спроса и предложения мощности (статическая реконфигурация), например, как показано в работах $[14,15]$.

Как правило, реконфигурация электрической сети для оптимизации параметров режима и снижения потерь электроэнергии имеет практический смысл с периодичностью до двух раз в сутки и не всегда оправдана с экономической точки зрения, чего нельзя сказать о реконфигурации для исключения перегрузки электрооборудования и восстановлении нормального режима при аварийных возмущениях.

Определение новой топологии электрической сети при динамической реконфигурации осуществляется на основе решения задачи (2) методом ветвей и границ при соблюдении условий допустимости режима (3), (4). Метод ветвей и границ базируется на следующих процедурах: задание исходного множества вариантов перебора, выбор наиболее перспективных множеств при разбиении исходного множества, ветв- 
ление перспективных множеств на подмножества перебора [16]. Для каждой границы определяется нижняя граница целевой функции, а для конечной вершины - точное значение минимизируемой целевой функции. Принцип ветвления заключается в обязательном участии и неучастии в дереве какой-либо ветви, т. е. исходное множество допустимых решений разбито на два непересекающихся подмножества: векторы с фиксированным значением 1 и 0 . При этом для каждого ветвления дерева электрической сети потери мощности вычисляются рекурсивно по представленным в (1) выражениях без расчета нелинейных уравнений установившихся режимов.

Некоторыми авторами при поиске точки потокораздела электрической сети используется подход, основанный на определении знака потоков мощности в ветвях схемы $[16,17]$ с полным их перебором. При этом возникает необходимость в дополнительных преобразованиях схемы электрической сети для исключения тупиковых ответвлений ветвей.

В соответствии с представленным на рис. 3 алгоритмом, если исключение перегрузки за счет реконфигурации невозможно по условиям допустимости режима (3), то осуществляется управление спросом и предложением мощности (возобновляемые источники энергии, накопители) в электрической сети вплоть до отключения нагрузки.

В качестве предложения мощности рассматриваются источники реактивной мощности и распределенной генерации, накопители (при наличии).

В данном случае под перегрузкой линий понимается превышение не предельной токовой нагрузки для конкретных типов проводов, а предельных значений токов с учетом обеспечения статической устойчивости и минимально необходимого резерва мощности [13].

Наряду с ограничением мощности потребителей в аварийных режимах соответствующей автоматикой (отключением) предлагается подход с более тщательным ранжированием потребителей с точки зрения возможного ущерба (стоимости) их отключения в реальном времени и их гибкости в управлении спроcom.

Подробнее рассмотрим подходы к снижению перегрузки элементов распределительной электрической сети по активной мощности, т. к. баланс реактивной мощности может быть обеспечен установленными устройствами компенсации реактивной мощности (отдельная задача за пределами данного исследования).

Основные способы снижения перегрузки линий:

- регулирование активной мощности генератора, солнечной электростанции, накопителя (при наличии);

- регулирование фазового угла (при технической возможности);

- настройка графика транзита мощности (импорт/экспорт);

- изменение топологии электрической сети;

- сброс нагрузки потребителей;
- у управление спросом активных потребителей. Сброс нагрузки является последним вариантом, когда перегрузка линий не может быть уменьшена другими методами.

В рамках реализации распределенного агентного управления потоками мощности в электрической сети [18] при перегрузке предлагаются следующие этапы разрешения указанной проблемы:

- предупреждение (наличие потенциальных условий для возникновения перегрузки с учетом прогнозных значений, структуры балансов мощности и прочих факторов);

- оповещение (превышение установленных динамических пороговых значений);

- аварийный режим;

- исключение перегрузки.

Регулирование активной мощности возобновляемых источников электроэнергии, накопителя осуществляется на основе оптимального выбора управляющих воздействий с учетом весовых коэффициентов и стоимости приращений генерации и изменения нагрузки и подробнее представлено в работе [17].

В качестве основного метода управляемого отключения потребителей при перегрузке элементов электрической сети предлагается использовать чувствительность потоков мощности в линиях к изменению нагрузки на шинах и приоритет нагрузки для указания важности нагрузки в какой-то момент времени.

Значения чувствительности на шинах нагрузки используются при расчете необходимого сокращения мощности для снижения перегрузки в линиях электрической сети.

Чувствительность перегруженной линии $i j$ для шины $k$ рассчитывается по формуле:

$$
\begin{gathered}
w_{i j}^{k}=\frac{\Delta P_{i j}}{\Delta P_{k}} ; \\
\Delta P_{i j}=P_{i j}-P_{i j}^{\max } .
\end{gathered}
$$

Нагрузка после сокращения на величину $\Delta P_{k}$ на шине $k$ для снижения перегрузки на $\Delta P_{i j}$

$$
P_{k}^{\prime}=P_{k}-\frac{w_{i j}^{k}}{\sum w_{i j}} \Delta P_{i j},
$$

где $P_{k}-$ исходная мощность нагрузки на шине $k$; $\Sigma w_{i j}$ - сумма чувствительностей по всем шинам для перегруженной линии $i j$.

Чем выше чувствительность $w_{i j}^{k}$, тем больше эффект от изменения мощности нагрузки на шине $k$ для снижения перегрузки в линии $i j$, рассчитываемый по (6).

Точный метод расчета чувствительности $w_{i j}^{k}$ может быть основан на известных соотношениях в матричной форме, связанных с топологией электрической сети [17].

Важность нагрузки в какой-то момент времени $t$ выражается в виде безразмерной величины и задается на основе экспертных оценок $\left(0<p_{t}<1\right)$. 
Приоритет нагрузки определяется путем ранжирования потребителей по важности нагрузки $p_{t}$. Отключение мощности потребителей для снижения перегрузки линий осуществляется по возрастанию важности $p_{t}$.

Активные потребители должны иметь возможность и желание снижать потребляемую мощность в период высоких цен на электроэнергию.

Многие авторы управление спросом активных потребителей рассматривают исходя из экономических интересов генерации, распределения и потребления электроэнергии, развивая различные рыночные модели их взаимодействия $[19,20]$.

Считаем, что управление спросом активных потребителей может осуществляется с учетом следующих факторов:

- чувствительность перегрузки линии к изменению нагрузки потребителей;

- ценовая характеристика нагрузки;

- приоритет нагрузки.

Чувствительность перегрузки линии к изменению нагрузки на шине $k$ для перегруженной линии $i j$ представлена в формуле (5). Для активных потребителей целесообразно ввести новый фактор чувствительности $\Delta w_{i j}^{k}$, который будет определяться близостью текущего $w_{i j}^{k}$ к средневзвешенному значению чувствительности $w s r_{i j}$ с учетом распределения мощности нагрузки в электрической сети:

$$
\begin{aligned}
\Delta w_{i j}^{k} & =w_{i j}^{k}-w s r_{i j} ; \\
w s r_{i j} & =\frac{\sum_{k}\left(w_{i j}^{k} P_{k}\right)}{\sum_{k} P_{k}} .
\end{aligned}
$$

В качестве ценовой характеристики нагрузки для активных потребителей может выступать величина превышения цены за электроэнергию $\Delta c^{k}$ над средневзвешенным значением $c s r$ какой-то момент времени:

$$
\begin{gathered}
\Delta c^{k}=c^{k}-c s r ; \\
c s r=\frac{\sum_{k}\left(c^{k} P_{k}\right)}{\sum_{k} P_{k}} .
\end{gathered}
$$

Нагрузка с высоким значением $\Delta c^{k}$ будет сокращена в первую очередь, т. к. для активного потребителя это значительный стимул для снижения электропотребления. С технической точки зрения наибольший результат для снижении мощности будет определяться активной нагрузкой с наибольшим значением чувствительности, рассчитываемым по выражению (7).

Для активных потребителей должны быть согласованы уровни сокращения нагрузки $\Delta P_{k \max }$ и $\Delta P_{k \min }$. В этом случае для потребителя является приемлемым участие в снижении перегрузки $\Delta P_{i j}$, если

$$
\Delta P_{k \min } \leq \frac{\Delta P_{i j}}{w_{i j}^{k}} \leq \Delta P_{k \max } .
$$

В целом целевая функция спроса для активных потребителей в $t$-й этап времени на основе выражения (8) может быть записана в виде:

$$
F\left(x_{t}\right)=\sum_{k} p_{t k} \Delta c_{t}^{k} x_{t k}=\sum_{k} G_{t k} x_{t k} \rightarrow \max
$$

при условии $\sum_{k} P_{t k} x_{t k} \leq P_{t \max }$,

где $x_{t k}$ - двоичная переменная (0 или 1); $G_{t k}=p_{t k} \Delta c_{t}^{k}$; $P_{t \max }-$ ограничение по мощности.

Согласно методу множителей Лагранжа, выражение (9) можно представить в виде:

$$
\begin{gathered}
L=F(x)-\sum_{k=1}^{n} \lambda_{k} P_{k}(x)=\sum_{k}\left\{G_{k} x_{k}-\lambda\left[P_{k} x_{k}-P_{\max }\right]\right\}= \\
=\sum_{k}\left(\varphi_{k} x_{k}+\lambda P_{\max }\right) ; L \rightarrow \max ,
\end{gathered}
$$

где $\varphi_{k}=G_{k}-\lambda P_{k}$

В Российской Федерации, в соответствии с Постановлением Правительства РФ от 20.07.2016 № 699 «О внесении изменений в Правила оптового рынка электрической энергии и мощности», получило развитие ценозависимое снижение потребления крупными потребителями оптового рынка электроэнергии и мощности, а также розничного рынка электроэнергии с участием организаций-агрегаторов.

Вовлечение потребителей розничного рынка в управление спросом на электроэнергию отсутствует. Представленная модель управления спросом активных потребителей, описываемая (9), (10), наряду с совершенствованием информационного обеспечения на базе технологий интернета вещей, может служить основой для построения перспективных управляемых распределительных электрических сетей.

\section{Практическая часть}

Рассмотрим реализацию представленных подходов к реконфигурации электрической сети 10 кВ (рис. 2). Основные параметры воздушных линий и расчетные нагрузки представлены в табл. 1, 2.

Таблица 1. Сопротивления линий

Table 1. Line resistances

\begin{tabular}{|c|c|c|c|c|c|c|c|}
\hline Линия/Line & 1 -1 & $2-3$ & $3-14$ & $4-5$ & $6-7$ & $8-9$ & $9-2$ \\
\hline$r(0) / O H)$ & 0,329 & $0-266$ & 1119 & 0,419 & 0,464 & 0,354 & 0,291 \\
\hline
\end{tabular} \begin{tabular}{|c|c|c|c|c|c|c|c|}
\hline$r(\mathrm{OM} / \mathrm{Ohm})$ & 0,329 & 0,266 & 1,119 & 0,419 & 0,464 & 0,354 & 0,291 \\
\hline$r(\mathrm{Om} / \mathrm{Ohm})$ & 0,099 & 0,080 & 0,144 & 0,102 & 0,113 & 0,064 & 0,087 \\
\hline
\end{tabular} \begin{tabular}{|c|c|c|c|c|c|c|c|}
\hline$x(\mathrm{OM} / \mathrm{Ohm})$ & 0,099 & 0,080 & 0,144 & 0,102 & 0,113 & 0,064 & 0,087 \\
\hline
\end{tabular} \begin{tabular}{|l|l|l|l|l|l|l|l}
\hline Линия/Line & $3 `-10$ & $11-12$ & $13-14$ & $15-16$ & $17-18$ & $18-19$ & $18-4$ \\
\hline
\end{tabular} \begin{tabular}{|c|c|c|c|c|c|c|c|}
\hline$r(\mathrm{OM} / \mathrm{Ohm})$ & 0,253 & 0,160 & 0,160 & 0,384 & 0,278 & 0,222 & 0,253 \\
\hline
\end{tabular} \begin{tabular}{|l|l|l|l|l|l|l|l|}
\hline$x(\mathrm{OM} / \mathrm{Ohm})$ & 0,076 & 0,039 & 0,039 & 0,094 & 0,084 & 0,040 & 0,076 \\
\hline
\end{tabular}

Таблица 2. Расчетные нагрузки

Table 2. Design loads

\begin{tabular}{|c|c|c|c|c|c|c|c|c|c|c|}
\hline $\begin{array}{c}\text { Нагрузка } \\
\text { Load bus }\end{array}$ & 1 & 2 & 3 & 4 & 5 & 6 & 7 & 8 & 9 & 10 \\
\hline P (кВт/kW) & 1200 & 930 & 1270 & 1100 & 820 & 910 & 830 & 560 & 780 & 860 \\
\hline Q (квар/kVar) & 1100 & 870 & 1140 & 930 & 620 & 820 & 730 & 420 & 650 & 760 \\
\hline $\begin{array}{c}\text { Нагрузка } \\
\text { Load bus }\end{array}$ & 11 & 12 & 13 & 14 & 15 & 16 & 17 & 18 & 19 & - \\
\hline P (кBт/kW) & 760 & 370 & 1200 & 1270 & 560 & 715 & 810 & 1100 & 390 & - \\
\hline Q (квар/kVar) & 650 & 290 & 1150 & 1100 & 430 & 550 & 725 & 970 & 300 & - \\
\hline $\begin{array}{c}\text { Генерация } \\
\text { Generation bus }\end{array}$ & 3 & 4 & 5 & 6 & 7 & 8 & 12 & 13 & 14 & 15 \\
\hline P (кВт/kW) & - & - & - & 150 & - & - & - & - & - & - \\
\hline Q (квар/kVar) & 400 & 400 & - & - & - & - & - & - & - & 200 \\
\hline
\end{tabular}


Моделирование рассматриваемой распределительной электрической сети выполнялось на имитационной модели в Matlab Simulink. Нагрузка задавалась с учетом типовых промышленно-бытовых статических характеристик по напряжению.

Для предварительной оценки снижения уровня потерь на участке электрической сети в результате реконфигурации был исследован участок с центрами питания ЦП 1 и ЦП 2, исключая ветвь 3-14 (рис. 2). Результаты имитационного моделирования с определением потребляемых мощностей по центрам питания ЦП 1 и ЦП 2 при различных местах потокораздела $\left(d_{i j}=0: 1-2,2-3,3-4,4-5,5-6,6-7,7-8,8-9\right)$ представлены на рис. 4, 5 .

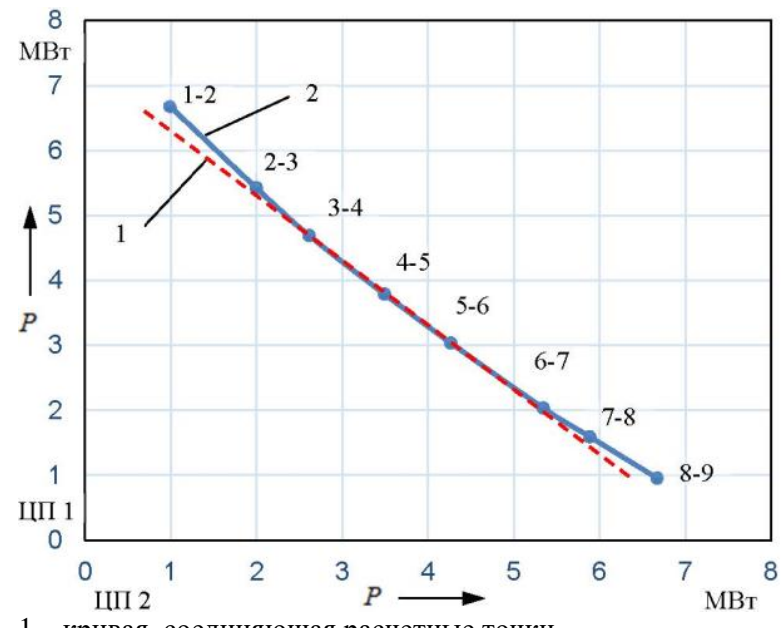

1 - кривая, соединяющая расчетные точки

2 - касательная к кривой 1 в точке 4-5

Puc. 4. Потребление мощњости центрами питания ЦП 1 и ЦП 2 при разделении электрической сети на два участка с раздельным питанием

Fig. 4. Power consumption by the power centers of ЦП 1 and ЦП 2 when the electrical network is divided into two sections with separate power supply

Как следует из рис. 4, минимальное потребление мощности от ЦП 1 и ЦП 2 достигается при раздельном питании рассматриваемого участка электрической сети с местом потокораздела $4-5\left(\delta_{45}=0\right)$. Очевидно, что перераспределение нагрузки в какой-то момент времени между шинами может привести к изменению оптимального места потокораздела.

В соответствии с представленным на рис. 3 алгоритмом для электрической схемы с четырьмя центрами питания, в результате решения оптимизационной задачи реконфигурации (2) были получены оптимальные места размыкания линий и количественные оценки параметров режима (табл. 3).

Цель реконфигурации - исключение перегрузки линий 1'-1 и 9-2' (менее 90 \%).

Реконфигурация электрической сети для быстрого снятия перегрузки элементов осуществляется на основе агентного подхода (подробнее в [18]) путем решения оптимизационной задачи (2) методом ветвей и границ с использованием рекуррентных соотношений для определения потерь электроэнергии для всех участков без расчета установившегося режима.

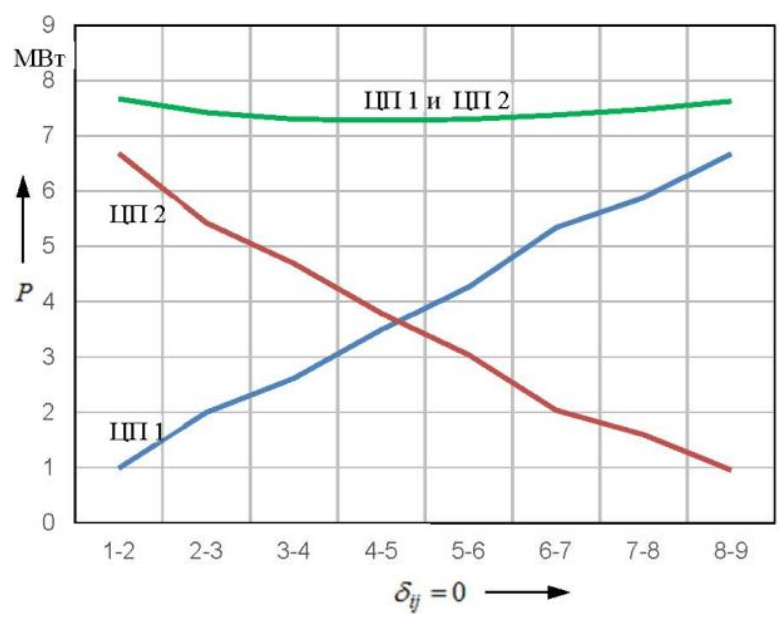

Puc. 5. Потребление мощности центрами питания ЦП 1 и ЦП 2 при различных местах потокораздела

Fig. 5. Power consumption by the power centers of ЦП 1 and ЦП 2 at various points in the flow area

Таблица 3. Потребление мощности в электрической сети до и после реконфигурации

Table 3. Power consumption in the electrical network before and after reconfiguration

\begin{tabular}{|c|c|c|c|c|c|c|}
\hline \multirow{3}{*}{$\begin{array}{c}\text { Режим элек- } \\
\text { трической } \\
\text { сети } \\
\text { Electric } \\
\text { network mode }\end{array}$} & \multicolumn{6}{|c|}{$\begin{array}{l}\text { Потребление мощности } \\
\text { Power consumption }\end{array}$} \\
\hline & \multirow{2}{*}{$\begin{array}{l}\text { активная } \\
\text { реактивная } \\
\text { active } \\
\text { reactive }\end{array}$} & \multicolumn{5}{|c|}{ ЦП/Power center } \\
\hline & & 1 & 2 & 3 & 4 & \\
\hline \multirow{2}{*}{$\begin{array}{c}\text { Исход- } \\
\text { ный/Original } \\
\delta_{34}, \delta_{1213}, \delta_{1415}=0\end{array}$} & $\mathrm{P}(\mathrm{MBT} / \mathrm{MW})$ & 225 & 1 & 343 & 4,1 & \\
\hline & Q (Мвар/MVar) & 12 & 88 & 011 & 3,5 & 1 \\
\hline \multirow{2}{*}{$\begin{array}{c}\text { После рекон- } \\
\text { фигурации } \\
\text { After } \\
\text { reconfiguration } \\
\delta_{45}, \delta_{314}, \delta_{1415}=0\end{array}$} & W) & 5,017 & 4,064 & 4,688 & 3,809 & 17 \\
\hline & Q (Мвар/MVar) & 4,447 & 3,59 & 4,463 & 3,344 & 15,844 \\
\hline
\end{tabular}

Из представленных в табл. 3 данных следует, что после реконфигурации потребляемая мощность в электрической сети снизилась на $2,9 \%$.

На рис. 6 представлены результаты имитационного моделирования напряжений на шинах электрической сети, на рис. 7, 8 - загрузка линий и потери мощности в них.

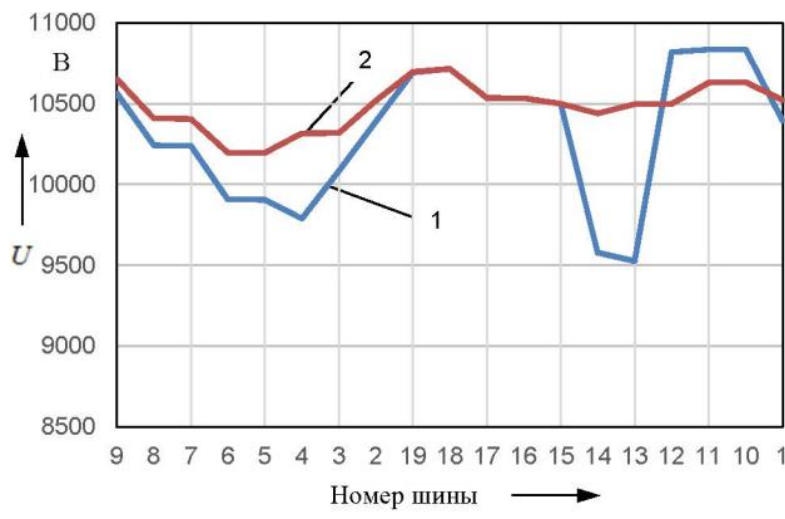

Puc. 6. Напряжения на шинах электрической сети до (линия 1) и после (линия 2) реконфигурации

Fig. 6. Voltages on electrical bus before (line 1) and after (line 2) reconfiguration 
Известия Томского политехнического университета. Инжиниринг георесурсов. 2020. Т. 331. № 3. 112-122

Черемисин В.Т., Третьяков Е.А. Повышение пропускной способности распределительных электрических сетей с ...

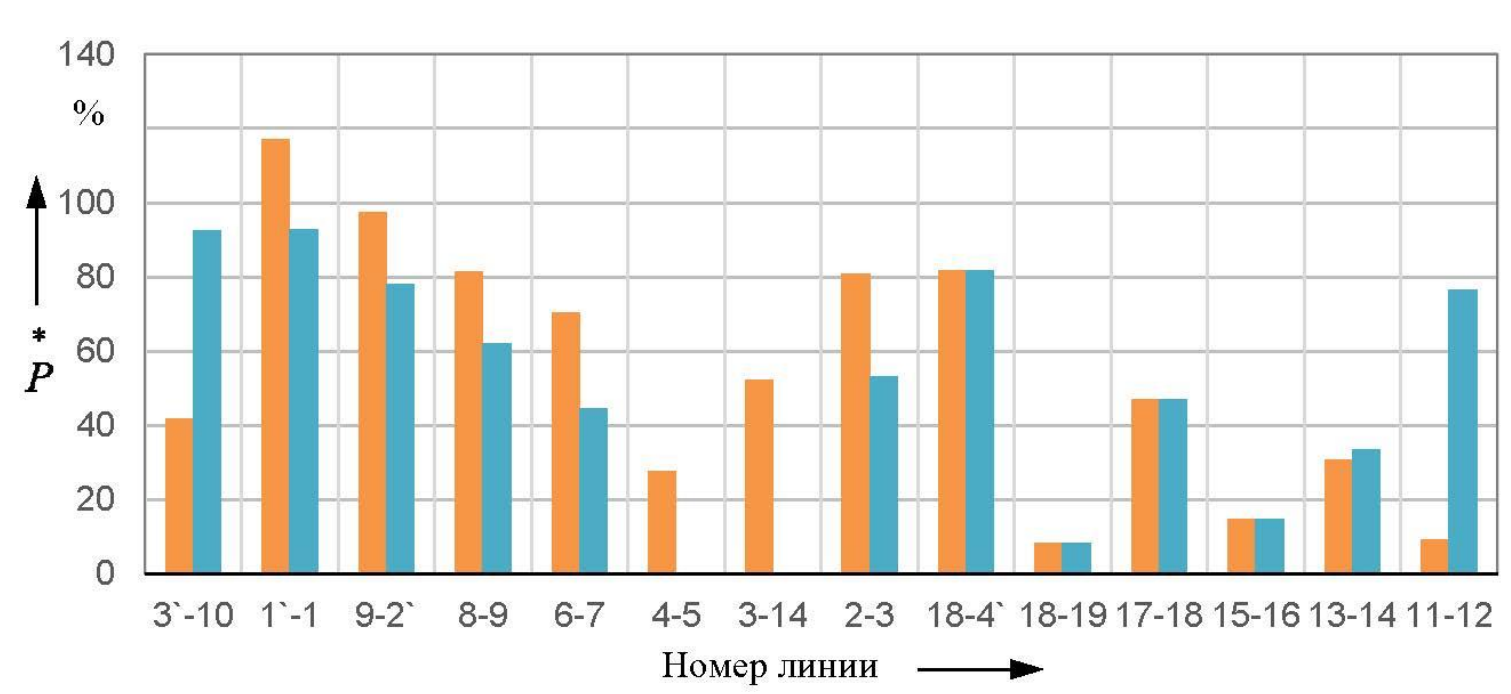

Рис. 7. Загрузка линий электрической сети до (левый столбеи) и после (правый столбеи) реконфигурации Fig. 7. Loading of electric lines before (left column) and after (right column) reconfiguration

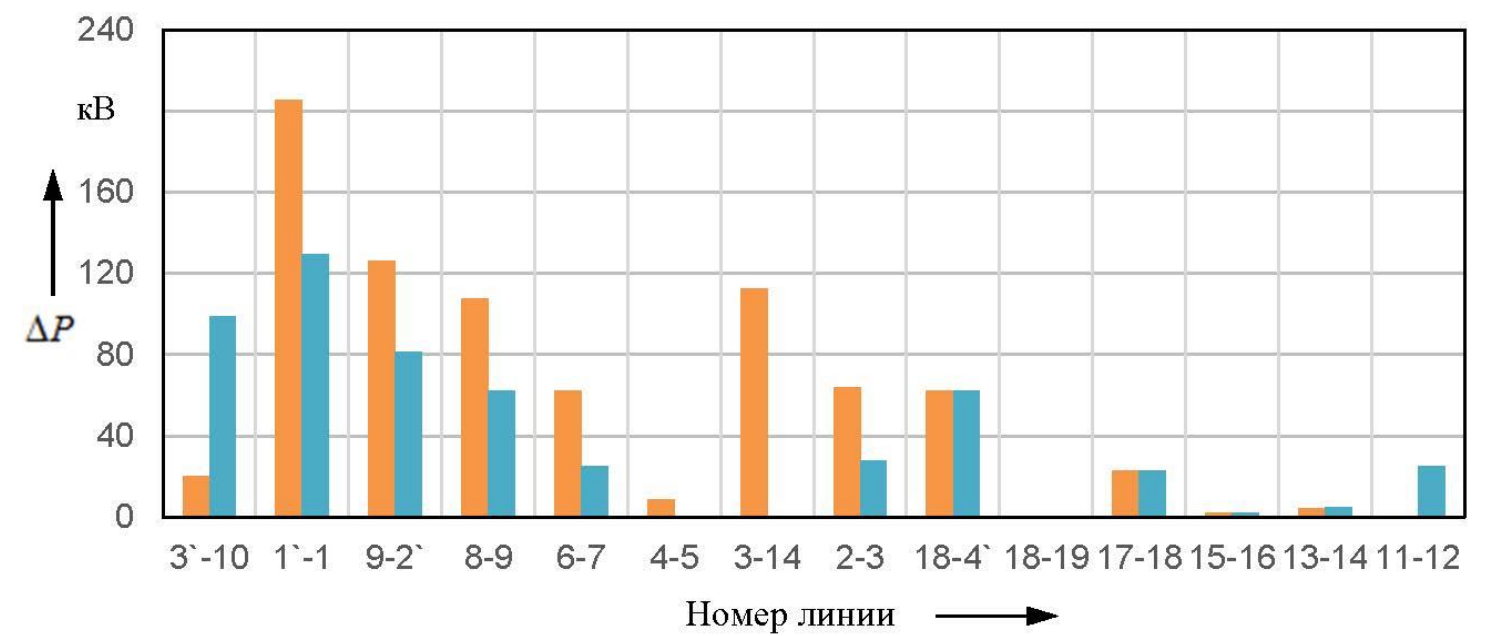

Рис. 8. Потери активной мощности в линиях электрической сети до (левый столбеи) и после (правый столбеи) реконфигурации

Fig. 8. Losses of active power in power lines before (left column) and after (right column) reconfiguration

Общие нагрузочные потери активной мощности в линиях электрической сети составили: 798,08 кВт до реконфигурации и 541,9 кВт после.

Как указывалось ранее, загрузка линий определяется с учетом запаса для обеспечения статической устойчивости (принято $10 \%$ и и минимально необходимого резерва мощности (при необходимости, в том числе по условию $n-1)$.

Таким образом, оптимальная реконфигурация для рассматриваемого случая обеспечила как допустимость режима по напряжению (рис. 5), так и исключение перегрузки линий. Поэтому в данном случае нет необходимости прибегать к ограничению мощности нагрузок и управлению спросом активных потребителей, что является предметом исследований авторов в других работах.

\section{Заключение}

Разработан алгоритм реконфигурации электрической сети для повышения ее пропускной способности на основе решения задач оптимизации нормальных режимов (статическая реконфигурация) и минимизации потребления электроэнергии без расчета установившихся режимов в темпе процессов изменения спроса на электроэнергию (динамическая реконфигурация). При этом в качестве критерия оптимизации топологии электрической сети выступает минимальное потребление электроэнергии при эксплуатационных ограничениях по схемам электроснабжения, допустимым параметрам режима, перегрузкам линий и т. д.

Представлены подходы к повышению пропускной способности электрической сети, если в результате реконфигурации не обеспечивается допустимый режим, за счет управления спросом, в том числе активных потребителей.

Практическая ценность представленных подходов заключается в том, что реконфигурация тестовой схемы позволила повысить пропускную способность электрической сети за счет балансировки нагрузок между линиями, устранить перегрузку линий и снизить потери мощности на $32 \%$. 
Достоверность полученных результатов подтверждается корректным использованием математического аппарата, известных положений фундаментальных и прикладных наук, сходимостью полученных теоретических результатов с данными вычислительных экспериментов.

\section{СПИСОК ЛИТЕРАТУРЬ}

1. Williams H.P. Logic and integer programming. - Boston: Springer 2009. $-464 \mathrm{p}$.

2. Aman M.M., Jasmon G.B., Naidu K. Discrete evolutionary programming to solve network reconfiguration problem // Proc. of the TENCON Spring Conference. - Sydney, Australia, 17-19 April 2013. - P. 23-29.

3. Manju M., Leena G., Saxena N.S. Distribution network reconfiguration for power loss minimization using bacterial foraging optimization algorithm // Engineering and Manufacturing. - 2016. - № 2. - P. 234-239.

4. Parallel power system restoration planning using heuristic initialization and discrete evolutionary programming / D.N.A. Talib, H. Mokhlis, M.S.A. Talip, K. Naidu // Modern Power Systems and Clean Energy. - 2017. - № 5. - Iss. 6. P. 991-1003.

5. Optimal reconfiguration based dynamic tariff for congestion management and line loss reduction in distribution networks / S. Huang, Q. Wu, L. Cheng, Z. Liu // IEEE Transactions on Smart Grid. - 2015. - № 7 (3). - P. 1295-1303.

6. Node depth encoding and multi objective evolutionary algorithm applied to large scale distribution system reconfiguration / A.C. Santos, C.B. Delbem, B.A. London, N.G. Bretas // IEEE Transactions on Power Systems - 2010. - № 25 (3). - P. 1254-1265.

7. Niknam T. An efficient hybrid evolutionary algorithm based on PSO and ACO for distribution feeder reconfiguration // European Transactions on Electrical Power. - 2010. - № 20. - P. 575-590.

8. Optimal reconfiguration of distribution system connected with distributed generations: a review of different methodologies / B. Ola, M. Saad, M. Hazlie, W. Dahalanc // Renewable and Sustainable Energy Reviews. - 2017. - № 73. - P. 854-867.

9. Mostafa S., Reza B. Optimal multi-objective reconfiguration and capacitor placement of distribution systems with the hybrid big bang - big crunch algorithm in the fuzzy framework // Shams Engineering Journal. - 2016. - № 7. - P. 113-129.

10. Гребенюк Г.Г., Крыгин А.А. Предельные графы в структурной оптимизации режимов распределительных сетей // Автоматика и телемеханика. - 2015. - № 1. - Р. 147-162.

11. Diestel R. Graph Theory. - New York: Springer-Verlag, 2005. $422 \mathrm{p}$.

12. Фишов А.Г., Мукатов Б.Б. Реконфигурация электрических сетей с распределенной генерацией и мультиагентным управлением // Известия Томского политехнического университета. Инжиниринг георесурсов - 2015. - Т. 326. - № 9. - С. 143-152.
Предлагаемые подходы к повышению пропускной способности распределительных электрических сетей могут найти применение в перспективных системах электроснабжения железных дорог с активно-адаптивной сетью, в изолированных маломощных микрогрид в рамках обособленных электротехнических комплексов [21, 22].

13. Веников В.А. Электрические системы. Электрические сети. М.: Высшая школа, 1998. - 511 с.

14. Выбор структуры электрических сетей промышленных предприятий в условиях автоматизированного управления электропотреблением / Д.В. Лукьянов, Д.А. Васильев, В.А. Иващенко, Д.В. Лукьянов, А.Ю. Шабельникова // Известия Вузов. Поволжский регион. Технические науки. - 2010. - № 2. C. 52-61.

15. Reza J.-S., Seyed-Masoud M.-T., Seyed-Sattar M. Microgrid operation and management using probabilistic reconfiguration and unit commitment // Electrical Power and Energy Systems. 2016. - № 75. - P. 328-336.

16. Peng Q., Low S.H. Optimal branch exchange for feeder reconfiguration in distribution networks // Decision and Control (CDC): IEEE 52nd Annual Conference on IEEE. - Italy, 2013. P. 2960-2965.

17. Jizhong Z. Optimization of power system operation - Piscataway: IEEE Press, 2009. - 624 p.

18. Перспективные методы управления транспортом и распределением электроэнергии в электрических сетях железных дорог / Е.А. Третьяков, Г.Е. Головнев, А.Г. Галкин, Е.А. Сидорова // Известия Транссиба. - 2018. - № 2 (34). - С. 113-124.

19. Развитие технологий активного потребителя и их интеграция в электрическую сеть общего пользования / Ю.Н. Кучеров, А.В. Иванов, Д.А. Корев, Н.А. Уткин, А.З. Жук // Энергетическая политика. - 2018. - № 5. - С. 73-86.

20. Arefi A., Abeygunawardana A., Ledwich G. A new risk-managed planning of electric distribution network incorporating customer engagement and temporary solutions // IEEE Transactions on Sustainable Energy. - 2018. - № 7 (4). - P. 1646-1661.

21. Балабанов М.С., Бабошкина С.В., Хамитов Р.Н. Экологические аспекты в энергосберегающей политике на этапе создания в России интеллектуальных энергосистем с активноадаптивной сетью // Известия Томского политехнического университета. Инжиниринг георесурсов. - 2015. - Т. 326. № 11. - С. 141-152.

22. Архипова О.В., Ковалев В.З., Хамитов Р.Н. Методика моделирования регионально обособленного электротехнического комплекса // Известия Томского политехнического университета. Инжиниринг георесурсов. - 2019. - Т. 330. - № 1. C. $173-180$

Поступила 22.02.2020 г.

\section{Информация об авторах}

Черемисин В.T., доктор технических, заведующий кафедрой подвижного состава электрических железных дорог, директор научно-исследовательского института энергосбережения на железнодорожном транспорте Омского государственного университета путей сообщения.

Tpemьяков E.A., кандидат технических наук, доцент кафедры подвижного состава электрических железных дорог Омского государственного университета путей сообщения. 


\title{
UDC 629.423 .1 \\ INCREASE OF PASSAGE CAPACITY OF DISTRIBUTIVE ELECTRIC NETWORKS WITH RENEWABLE ENERGY SOURCES THROUGH THEIR RECONFIGURATION
}

\author{
Vasily T. Cheremisin 1 , \\ cheremisinvt@gmail.com
}

\author{
Evgeny A. Tretyakov 1 , \\ eugentr@mail.ru
}

1 Omsk State Transport University,

35, K. Marx avenue, Omsk, 644046, Russia.

\begin{abstract}
The relevance of the study is caused by the need to develop technical solutions to increase the capacity of promising distribution electric networks of railways with renewable energy sources in the pace of changing demand for electricity.

The aim of research is to increase the capacity of promising distribution electric networks of railways with renewable energy sources through optimal reconfiguration of electrical circuits.

Objects: distribution electric networks, sources of distributed generation of active and reactive power, optimization methods for solving problems in the electric power industry, increasing the capacity of the electric network.

Methods. Optimization problem was solved by the branch and bound method, simulation modeling of the electrical network modes was performed in Matlab Simulink.

Results. The authors have developed the algorithm for reconfiguring the electrical network to increase its capacity based on solving the problems of optimizing normal modes (static reconfiguration) and minimizing power consumption without calculating steady-state modes in the pace of electricity demand change processes (dynamic reconfiguration). To ensure the admissibility of the modes of distribution electric networks, approaches to managing the supply of active (based on renewable sources) and reactive capacities and the demand of active consumers were proposed. The paper introduces the results of implementation of dynamic reconfiguration for the test circuitry without taking into account the power supply and demand management of active consumers, indicating the validity of the proposed approaches to improving the transmission capacity of the electrical network.
\end{abstract}

\section{Key words:}

Reconfiguration, distribution electric networks, transmission capacity, sources of distributed generation of active and reactive power, demand management.

\section{REFERENCES}

1. Williams H.P. Logic and integer programming. Boston, Springer, 2009. $464 \mathrm{p}$.

2. Aman M.M., Jasmon G.B., Naidu K. Discrete evolutionary programming to solve network reconfiguration problem. Proceedings of the TENCON Spring Conference. Sydney, Australia, 17-19 April 2013. pp. 23-29.

3. Manju M., Leena G., Saxena N.S. Distribution network reconfiguration for power loss minimization using bacterial foraging optimization algorithm. Engineering and Manufacturing, 2016, no. 2, pp. 234-239.

4. Talib D.N.A., Mokhlis H., Talip M.S.A., Naidu K. Parallel power system restoration planning using heuristic initialization and discrete evolutionary programming. Modern Power Systems and Clean Energy, 2017, no. 5, Iss. 6, pp. 991-1003.

5. Huang S., Wu Q., Cheng L., Liu Z. Optimal reconfiguration based dynamic tariff for congestion management and line loss reduction in distribution networks. IEEE Transactions on Smart Grid, 2015 , no. 7 (3), pp. 1295-1303.

6. Santos A.C., Delbem C.B., London B.A., Bretas N.G. Node depth encoding and multi objective evolutionary algorithm applied to large scale distribution system reconfiguration. IEEE Transactions on Power Systems, 2010, no. 25 (3), pp. 1254-1265.

7. Niknam T. An efficient hybrid evolutionary algorithm based on PSO and ACO for distribution feeder reconfiguration. European Transactions on Electrical Power, 2010, no. 20, pp. 575-590.

8. Ola B., Saad M., Hazlie M., Dahalanc W. Optimal reconfiguration of distribution system connected with distributed generations: a review of different methodologies. Renewable and Sustainable Energy Reviews, 2017, no. 73, pp. 854-867.

9. Mostafa S., Reza B. Optimal multi-objective reconfiguration and capacitor placement of distribution systems with the hybrid big bang - big crunch algorithm in the fuzzy framework. Shams Engineering Journal, 2016, no. 7, pp. 113-129.
10. Grebenyuk G.G., Krygin A.A. Predelnye grafy v strukturnoy optimizatsii rezhimov raspredelitelnykh setey [Limit graphs in structural optimization of distribution network modes]. Automation and Remote Control, 2015, no. 1, pp. 147-162.

11. Diestel R. Graph Theory. New York, Springer-Verlag, 2005. 422 p.

12. Fishov A.G., Mukatov B.B. Reconfiguration of electrical networks with distributed generation and multi-agent control. Bulletin of the Tomsk Polytechnic University. Geo Assets Engineering, 2015, vol. 326, no. 9, pp. 143-152. In Rus.

13. Venikov V.A. Elektricheskie sistemy. Elektricheskie seti [Electrical systems. Electricity of the net]. Moscow, vysshaya shkola Publ., 1998. 511 p.

14. Lukyanov D.V., Vasilev D.A., Ivashchenko V.A., Lukyanov D.V., Shabelnikova A.Yu. Vybor struktury elektricheskih setey promyshlennykh predpriyatiy $\mathrm{v}$ usloviyakh avtomatizirovannogo upravleniya elektropotrebleniem [The choice of the structure of electrical networks of industrial enterprises in the conditions of automated power consumption management]. News of universities. Volga region. Technical science, 2010, no. 2, pp. 52-61.

15. Reza J.-S., Seyed-Masoud M.-T., Seyed-Sattar M. Microgrid operation and management using probabilistic reconfiguration and unit commitment. Electrical Power and Energy Systems, 2016, no. 75 , pp. $328-336$.

16. Peng Q., Low S. H. Optimal branch exchange for feeder reconfiguration in distribution networks. Decision and Control $(C D C)$. IEEE 52 ${ }^{\text {nd }}$ Annual Conference on IEEE. Italy, 2013. pp. 29602965.

17. Jizhong Z. Optimization of power system operation. Piscataway, IEEE Press, 2009. $624 \mathrm{p}$.

18. Tretyakov E.A., Golovnev G.E., Galkin A.G., Sidorova E.A. Perspective methods of transport management and distribution of electricity in electric networks of railways. Izvestiya Transsiba, 2018, no. 2 (34), pp. 113-124. In Rus.

19. Kucherov YU.N., Ivanov A.V., Korev D.A., Utkin N.A., Zhuk A.Z. The development of active consumer technologies and 
their integration into the public electrical network. Energy Policy, 2018, no. 5, pp. 73-86. In Rus.

20. Arefi A., Abeygunawardana A., Ledwich G. A new Risk-Managed Planning of Electric Distribution Network Incorporating Customer Engagement and Temporary Solutions. IEEE Transactions on Sustainable Energy, 2018, no. 7 (4), pp. 1646-1661.

21. Balabanov M.S., Baboshkina S.V., Khamitov R.N. Electric saving and environmental aspects in policy at the stage of smart grid crea- tion in Russia. Bulletin of the Tomsk Polytechnic University. Geo Assets Engineering, 2015, vol. 326, no. 11, pp. 141-152. In Rus.

22. Arkhipova O.V., Kovalev V.Z., Khamitov R.N. Methodology of modeling regionally isolated electrotechnical complex. Bulletin of the Tomsk Polytechnic University. Geo Assets Engineering, 2019, vol. 330, no. 1, pp. 173-180. In Rus.

Received: 22 February 2020.

\section{Information about the authors}

Vasiliy T. Cheremisin, Dr. Sc., head of the department, Director of the Energy Saving Research Institute, Omsk State Transport University.

Evgeny A. Tretyakov, Cand. Sc., associate professor, Omsk State Transport University. 